Prepared for the U.S. Department of Energy

under Contract DE-AC05-76RL01830

\title{
Progress Toward Producing Demand-Response-Ready Appliances
}

\author{
DJ Hammerstrom
}

C Sastry

December 2009

Pacific Northwest

NATIONAL LABORATORY

Proudly Operated by Battelle Since 1965 


\title{
DISCLAIMER
}

This report was prepared as an account of work sponsored by an agency of the United States Government. Neither the United States Government nor any agency thereof, nor Battelle Memorial Institute, nor any of their employees, makes any warranty, express or implied, or assumes any legal liability or responsibility for the accuracy, completeness, or usefulness of any information, apparatus, product, or process disclosed, or represents that its use would not infringe privately owned rights. Reference herein to any specific commercial product, process, or service by trade name, trademark, manufacturer, or otherwise does not necessarily constitute or imply its endorsement, recommendation, or favoring by the United States Government or any agency thereof, or Battelle Memorial Institute. The views and opinions of authors expressed herein do not necessarily state or reflect those of the United States Government or any agency thereof.

\author{
PACIFIC NORTHWEST NATIONAL LABORATORY \\ operated by \\ BATTELLE \\ for the \\ UNITED STATES DEPARTMENT OF ENERGY \\ under Contract DE-AC05-76RL01830 \\ Printed in the United States of America \\ Available to DOE and DOE contractors from the \\ Office of Scientific and Technical Information, \\ P.O. Box 62, Oak Ridge, TN 37831-0062; \\ ph: (865) 576-8401 \\ fax: (865) 576-5728 \\ email: reports@adonis.osti.gov

\footnotetext{
Available to the public from the National Technical Information Service, U.S. Department of Commerce, 5285 Port Royal Rd., Springfield, VA 22161 ph: (800) 553-6847 fax: (703) 605-6900 email: orders@ntis.fedworld.gov online ordering: http://www.ntis.gov/ordering.htm
} 


\title{
Progress Toward Producing Demand-Response-Ready Appliances
}

\author{
DJ Hammerstrom \\ C Sastry
}

December 2009

Prepared for

the U.S. Department of Energy

under Contract DE-AC05-76RL01830

Pacific Northwest National Laboratory

Richland, Washington 99352 



\begin{abstract}
This report summarizes several historical and ongoing efforts to make small electrical demand-side devices like home appliances more responsive to the dynamic needs of electric power grids. Whereas the utility community often reserves the term demand response for infrequent two- to six-hour curtailments that reduce total electrical system peak load, other beneficial responses and ancillary services may also be provided by responsive electrical demand. Historically, demand responses have been obtained by applying external, retrofitted, controlled switches to existing electrical demand. This report is directed instead toward manufactured products, including appliances, that are able to provide demand responses as soon as they are purchased and that require few, or no, after-market modifications to make them responsive to needs of power grids. Efforts to be summarized include Open Automated Demand Response, the Association of Home Appliance Manufacturers standard CHA-1, a simple interface being developed by the U-SNAPTM Alliance, various emerging autonomous responses, and the recent PinBus interface that was developed at Pacific Northwest National Laboratory.
\end{abstract}





\section{Acronyms and Abbreviations}

AHAM

AMI

ANSI

AutoDR

CHA-1

DRAS

GFA

HAN

LBNL

OpenADR

OSI

PCT

PNNL

RDS

U-SNAP

Z-Wave
Association of Home Appliance Manufacturers

advanced metering infrastructure

American National Standards Institute

Automated Demand Response

Connected Home Appliance standard, version 1

demand-response automation server

Grid Friendly ${ }^{\mathrm{TM}}$ Appliance

home area network

Lawrence Berkeley National Laboratory

Open Automated Demand Response

Open Systems Interconnection

programmable communicating thermostat

Pacific Northwest National Laboratory

Radio Data System

Utility Smart Network Access Port

a wireless-communications proprietary standard designed for home automation 



\section{Contents}

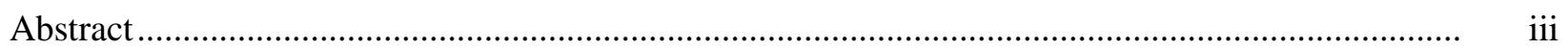

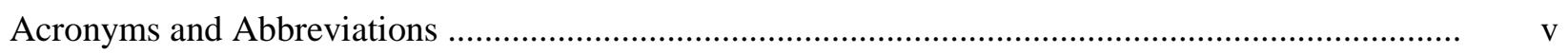

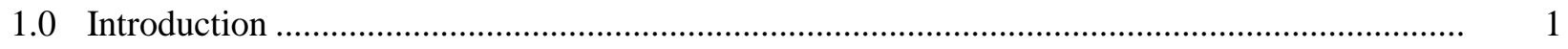

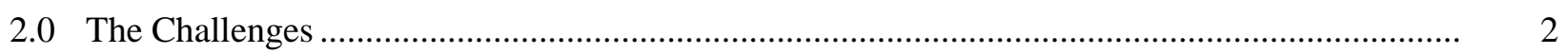

3.0 Available Devices ...................................................................................................

4.0 Standards and Activities that Encourage Demand-Response-Ready Device Development......... 4

4.1 Open Automated Demand Response (OpenADR) ...................................................... 5

4.2 Association of Home Appliance Manufacturers (CHA-1) ................................................ 7

4.3 Utility Smart Network Access Port (U-SNAP) ........................................................... 8

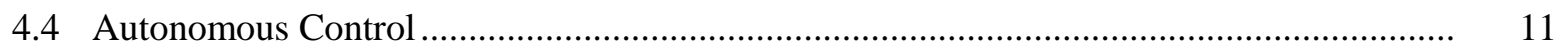

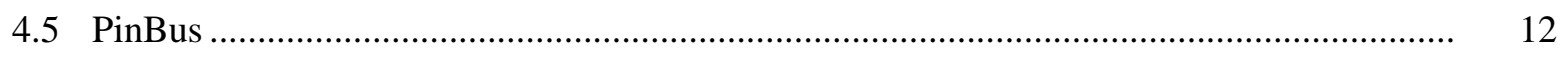

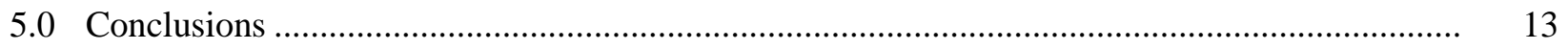

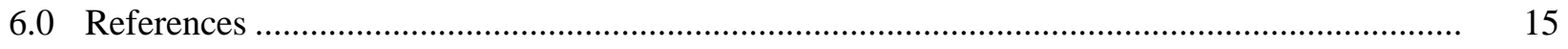

\section{Figures}

1 Architecture Illustrating the Communication between a DRAS and DRAS Clients .................. 5

2 CHA-1 Appliance Model Object Hierarchy ................................................................... 8

3 The U-SNAP Vision to Enable Communication between HAN Devices and an AMI Smart Meter is Agnostic of the Underlying Communication or Messaging Protocol........................... 9

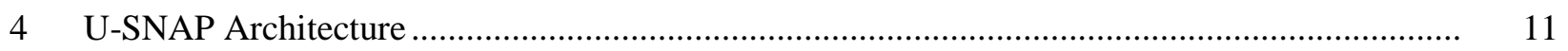

\section{Tables}

1 Capabilities that can be Communicated across the PinBus Interface with Various Numbers of Device Pins.....

2 Issues Addressed by the Standards and Approaches Discussed in this Paper..... 



\subsection{Introduction}

This report summarizes several efforts to simplify and reduce the costs, and thereby encourage the practice, of demand response. The term demand response will be applied quite broadly herein to refer to all the various power-grid objectives that may be accomplished by applying and controlling responsive electrical demand. The utility industry often uses the term demand response more narrowly to address the relatively long but infrequent curtailment of electrical loads that helps reduce total system peak electrical demand. However, more innovative and dynamic demand responses address other, perhaps ancillary, grid services. Very dynamic demand responses, for example, can modulate demand every few seconds to respond to a region's area-control error and thereby help control grid frequency.

This report will not directly address energy efficiency as a demand-response strategy. Energy efficiency is the reduction of the energy needed to accomplish a given task. As it is applied programmatically by utilities, energy efficiency is a form of demand response, but energy efficiency, in this limited sense, offers no dynamic responsiveness and will not be relevant to this report. Incidentally, electrical demand may be dynamically controlled to achieve significant system energy efficiency benefits, which response would indeed be relevant to this report.

Much of the expense now incurred by utility demand-response programs is spent on after-market equipment and the installation of such equipment by skilled technicians. The costs of performing demand response might be greatly reduced if devices such as appliances were designed and manufactured ready to respond to the grid or to signals that represent the needs of an electric power grid. Necessary product design modifications would then be made, and these modifications would be completed on manufacturing floors, where labor is relatively inexpensive. An interesting analysis of the consequent cost benefit (Eustis, Horst and Hammerstrom 2007) concluded that only demand responses made by central air conditioning now compete favorably against the installation of new combustion turbines, a recognized alternative to demand response. However, even relatively small appliances could compete favorably against new combustion turbines if the appliances were manufactured ready to respond to demandresponse needs or signals.

A preferred demand-response interface solution should be elegant and simple. These attributes put such approach at an advantage over disjoint or complex approaches.

Standardization will, of course, facilitate the adoption of winning demand-response interface approaches. Elegant and simple interface approaches can be more readily defined and standardized. Ultimately, standardization also reduces many of the expenses and risks of an engineered solution. Standardization is required at all levels, from the definition of the physical interface itself to the definition of signals and their semantics. The business approach, too, must be defined and standardized. It will be concluded in this report that the lack of business-practice standardization is the greatest present barrier to achieving a large resource of demand-responsive devices.

This report will list many of the challenges that must be addressed and will summarize some of the approaches and standards that have been proposed to create more, and more-demand-responsive, devices. Examples to be discussed will include Open Automated Demand Response (OpenADR ${ }^{\mathrm{TM}}$ ), the Association of Home Appliance Manufacturer (AHAM) Connected Home Appliance standard (CHA-1), a simple interface being developed by the Utility Smart Network Access Port (U-SNAPTM) Alliance, 
various emerging autonomous responses, and the PinBus interface that is being developed at Pacific Northwest National Laboratory.

\subsection{The Challenges}

This section presents motivation for a standardized interface for responsive demand. Several issues that presently limit the practice of demand response will be discussed; these issues should be reconsidered by the reader in the next sections, when several examples of interface standards will be discussed, to see how these challenges are mitigated by the various standards and approaches.

As has been stated, it is presently expensive to control a responsive device because the control must be applied through after-market, add-on control boxes, which additional equipment must often be installed by skilled technicians. The average expense of controlling devices may be reduced if each device is manufactured ready to respond. However, the small incremental costs of making each responsive device demand-response ready sums to a large total manufacturing investment. This large investment must still be paid ultimately by those who would benefit. Unfortunately, no benefit can be realized from the investment in those demand-response-ready appliances that are retailed but do not become engaged by any of the relatively sparse demand-response programs offered by utilities, independent system operators, and states.

Demand response is presently practiced regionally, whereas the devices that could perform such demand responses are manufactured more centrally and are targeted to customers in multiple regions and even internationally. Expensive modifications of automated manufacturing lines are not likely justified by utility and regional demand-response programs. If such products could be differentiated to accommodate multiple regional programs, product distribution practices too would need to be tailored to disperse the various product models to their respective regions. Consequently, potentially responsive devices are not readily modified to suit the requirements of individual states, regions, and utilities.

Standardization of demand responses should be initiated from the device side, not the utility side, of a device interface. Efforts to develop demand response have thus far been led by utilities, but responsive devices might also be made responsive to entertainment systems, premises-level energy-control systems, premises security systems, and other stakeholder entities. There are perhaps 1000 or so interfaces between a utility entity and its many substations, another 1000 or so customers for every substation, and up to ten potentially responsive devices within each premises. Approaching this from a system-engineering perspective, one should begin at the device interface, where the most interfaces and the most potential impact from reducing expenses would exist.

Standardization of a demand-response-ready program will require that a cohesive formulation of the problem must be completed across all interested business entities and throughout the entire communication chain that would be needed to make such devices responsive. Utilities, regulators, business owners, states, homeowners, and manufacturers must align themselves to complete the demandresponse application. Communication must be planned and executed all the way from the utility entity that might wish to control demand through long-haul communication pathways, into buildings, into communication gateways within premises, and finally into the responsive device. If required, information must flow upstream from devices back toward the utility entity, too. The failure of any stakeholder or 
communication link to fulfill its responsibility could compromise the entire application. Perhaps, we suggest, advocates of demand-response-ready devices should seek ways to decouple the stakeholders and communication links from one another, so that no one stakeholder or communication link can defeat an entire application.

Depending on one's perspective, intellectual property is either the solution or a blockade to development of demand-responsive devices for the smart grid. Those who have invested in devices and protocols naturally seek ways to be compensated for their investments. Those who are developing demand-response programs naturally wish to apply inexpensive technologies that are free of any encumbrances and further wish to have devices offered competitively by multiple vendors.

Another challenge is to accommodate historical demand-response programs and practices while the practice of demand response evolves. Prior demand-response programs and approaches have targeted specific types of responsive devices within limited geographic regions. The competing control products offered by the various vendors have been diverse. For example, competing practices have emerged and remain supported for both thermostatic set-point control and the duty cycling of air conditioners. Utilities (and regional operators) formulate different programmatic approaches because their circumstances differ. They have diverse customer types, different existing infrastructure, and different operational objectives. This degree of diversity at the device and programmatic levels especially challenges standardization efforts, which must also then accommodate most, if not all, existing and historical devices, practices and programmatic approaches. If practitioners of demand response are willing to consolidate these diverse approaches, perhaps abandoning marginal and dated approaches, simpler and more elegant approaches might emerge. For example, the authors have been advocating that high-level grid management objectives, not device-specific commands, should be communicated to responsive devices. This practice would potentially limit some demand-response approaches, but it would ultimately simplify communication to responsive devices.

The business cases for demand-response-ready devices are either incomplete or altogether absent. Utilities remain reluctant to commit to a uniform and standardized installation of demand-response-ready devices. It is reasonable that they would remain reluctant until their own business cases for doing so are proven within the utility. Manufacturers remain unable to commit to the manufacture of demandresponse-ready devices until their customers demand and will pay for such devices. Therefore, while the outcome of an investment in demand-response-ready approaches may be enticing, the steps to achieve this transformational approach are elusive. This condition creates a challenging technological and business stalemate.

The manufacturer of a responsive device that integrates digital communication or control equipment into his products risks tying the lifecycles of his products to those of the digital components. Appliances may function for decades, but digital communication and control equipment has demonstrated much shorter useful lifecycles. These digital components might become obsolete long before the appliance would otherwise fail. Newer network systems might not support dated devices. Repair and support services might become unavailable for dated devices for which the digital equipment is no longer supported. Therefore, the manufacturer must manage design decisions that could prematurely make its products obsolescent. 


\subsection{Available Devices}

This section lists some available device types that have been produced, or will soon be produced, ready to respond to demand-response signals. Noteworthy products include thermostats and some home appliances.

Programmable communicating thermostats (PCTs) are arguably among the first demand-responseready devices. Such thermostats have been offered by multiple businesses that provide demand-responseaggregation services. Additional vendors have benefited from California utilities' concerted efforts to apply these "smart" thermostats. The thermostat occupies a fortuitous position as a retrofittable controller of a major premises electrical load, the air conditioner. A moderate price premium can be justified and offered for thermostats that transmit signals wirelessly, or by power line, and can therefore participate in communicated demand-response requests. A thermostat connects to the rest of a premises' air conditioning system using safe signal voltages and can therefore be installed quite safely and by technicians having modest levels of training. The programmable communicating thermostat often communicates with a home gateway or advanced meter, which some might argue are also demandresponse-ready. We prefer to consider these latter devices as enabling devices, not responsive ones.

Appliances, including white-goods appliances, have been manufactured ready to respond to communicated signals. Communicating appliances are not new, but some major appliance manufacturers have recently announced product offerings on a large enough scale that they might become commercially successful. General Electric has announced the availability of a suite of responsive appliances; these products are presently being tested in field trials in Louisville, Kentucky (GE 2009). Whirlpool Corporation followed suit with a declaration that it would also offer communicating, responsive appliances by 2015 if a standard communication platform and means to recover such an investment becomes available in 2010 (Whirlpool 2009).

The fact that relatively few demand-response-ready devices are available emphasizes the challenge of producing and marketing such devices. As was pointed out in the challenges stated in Section 2.0, the production of viable demand-response-ready devices will succeed only after such devices are supported by standards, a critical mass of necessary infrastructure, and workable business cases.

\subsection{Standards and Activities that Encourage Demand- Response-Ready Device Development}

This section reviews progress that has been made toward the development of interfaces and relevant interface standards that could facilitate the production of demand-response-ready devices. The items to be discussed include

- OpenADR

- The AHAM CHA-1 standard

- U-SNAPTM

- Autonomous control

- PinBus. 


\subsection{Open Automated Demand Response (OpenADR)}

In the aftermath of the California electricity crisis in 2002, the Demand Response Research Center at Lawrence Berkeley National Laboratory (LBNL), with sponsorship from the California Energy Commission, undertook research and development that resulted in automated demand response (AutoDR) (LBNL 2009a). The motivations for AutoDR were the need to shed electrical demand and manage grid emergencies during periods of peak power demand. AutoDR links a facility's energy management systems with any from among recommended customer-programmed energy management strategies. These programmed control strategies initiate dimming or turning off of lights, reduction of air conditioning load in certain zones of the building, changed thermostat set points, and turning off of specific pieces of equipment like fans and humidifiers (LBNL 2009). As an adjunct benefit, a customer's process of identifying which loads to shed also helps them improve the overall energy efficiency of their buildings. Since customer loads are shed automatically, AutoDR achieves high levels of participation compared to non-automated demand-response strategies.

The demand response automation server (DRAS) within an AutoDR system is a centralized computer system that provides continuous signals to automate customers' reduction of power demand (Akuacom 2009, Koch and Piette 2007). DRAS clients are the automated agents at customer premises that receive and respond to signals from the DRAS. The architecture that facilitates AutoDR through a DRAS is shown below in Figure 1.

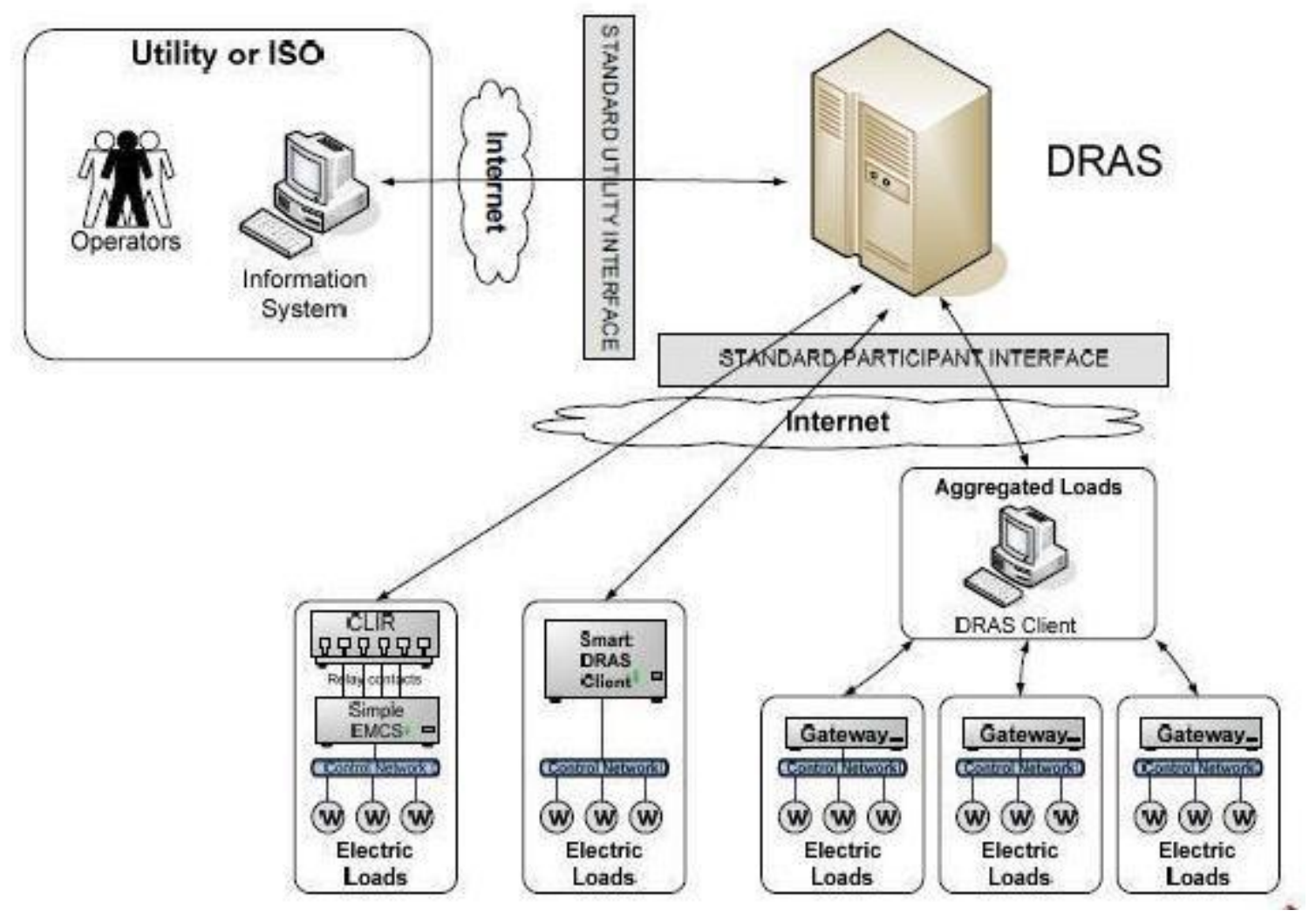

Figure 1. Architecture Illustrating the Communication between a DRAS and DRAS Clients (Koch and Piette 2007, p. 2) 
Whenever utilities, regional transmission organizations, or independent system operators require electrical load to be shed, demand-response-event notifications are published to DRAS clients at the premises of participating customers. The DRAS clients activate their preprogrammed strategies and load is shed. Facility managers receive alerts informing them of demand-response events and may intervene to reduce or increase a building's level of participation. These measures continue to be in place until the DRAS clients receive another notification returning the building to its normal behavior.

The communication between a DRAS and DRAS clients takes place through standard open protocols like extensible markup language (XML) and secure web-based service-oriented architecture (SOA). These open-standards-based communications and associated data models are formally specified as an application-level protocol standard, referred to as the Open Automated Demand Response Communications Specification, also known as OpenADR (LBNL 2009c). The open specification is intended to allow anyone to implement both the DRAS and DRAS clients. Akuacom simplified this process further through their OpenADR client development program (Akuacom 2009). The OpenADR specification is currently in the process of standardization through the Organization for the Advancement of Structured Information Standards (OASIS 2009), Utility Communication Architecture International Users Group (UCAIug 2009), and other interested stake holders through the OpenADR outreach collaborative (LBNL 2009b).

The following list highlights the major characteristics of the OpenADR application level protocol (McParland 2009):

- Continuous and reliable - provides a continuous, secure, and reliable two-way information exchange infrastructure in which the DRAS clients receive and acknowledge demand-response event signals from the DRAS

- Signal-based - translates price and event information into continuous five-minute Internet signals to facilitate automation of demand responses. These signals are designed to interoperate with facility energy-management and control systems, lighting controls, or other end-use control devices.

- Automated-receipt of a demand-response-event signal initiates an automated response via preprogrammed strategies that have been established by the end-use customer

- Overridable - provides an opt-out or override function to consumers if an event should come at a time when curtailments are unacceptable

- Complete - describes a rich, complete data model and architecture to communicate price, reliability, and other demand-response activation signals

- Scalable - provides an architecture that is scalable to different forms of demand-response programs and tariffs

- Standardized —open standards-based technology (such as simple object access protocol (SOAP)) and web services form the basis of the communication standard. Uses standardized server and client message formats and select extensible markup language tags to allow future semantic and content extensions to OpenADR messages.

AutoDR programs through OpenADR are currently targeted toward automating curtailment of large commercial and industrial customer loads. Some utilities that offer AutoDR programs to their commercial and industrial customers include Pacific Gas and Electric Company, Southern California 
Edison, San Diego Gas \& Electric Company, Global Energy Partners, LLC, and Bonneville Power Administration/Seattle City Light. OpenADR is also enabling a wholesale-market ("Ancillary Services Participating Load") implementation with the California Independent System Operator (CAISO) and Pacific Gas \& Electric (AutomatedBuildings 2009).

In summary, OpenADR is a standard for facilitating distributed demand-response. OpenADR helps building and facilities managers implement automated demand response in their facilities. Using OpenADR, electric utilities can help their commercial and industrial customers participate in powerpricing programs and AutoDR. Finally, OpenADR helps manufacturers of building-automation equipment design products for smart grid implementation and helps aggregators incorporate demand response into the services that they offer.

\subsection{Association of Home Appliance Manufacturers (CHA-1)}

AHAM is a U.S.-based trade association of the home-appliance manufacturing industry. Its members include the manufacturers of major, portable, and floor-care home appliances and these manufacturers' suppliers (AHAM 2009a). AHAM is an American National Standards Institute (ANSI 2009) member and an accredited standards development organization. AHAM has undertaken efforts to formulate interoperability standards for communication between home appliances and other devices; these standards could enable demand-response applications.

AHAM initially began to develop a complete standard that would facilitate the interoperability of smart appliances at the interface between the appliance and communication networks. Extensive discussions were held to complete this communications standard, including all seven layers of the Open System Interconnection (OSI) reference model, from the physical layer up through the application layer. The OSI model is a widely recognized standard framework for transmitting messages between two points within a telecommunications network. Later, the task force altered its course and opted for an approach that instead emphasized only the application layer of the OSI stack.

AHAM then completed the Connected Home Appliances standard, version 1 (CHA-1), which was based on the principles of object-oriented software engineering and design. The standard was later approved as an American National Standard in 2003 (AHAM 2009b). As with all AHAM standards, CHA-1 is an open, voluntary, consensus standard. It is available for use by anyone and imposes no licensing requirements. Most importantly, it is extensible by manufacturers of appliances, who may add customized features to their own version.

The CHA-1 standard is based on the object-oriented software design principle that includes objects, classes, methods, and events. Each AHAM appliance is modeled as a collection of objects-instantiations of abstract elements (classes) that include various appliance functions (methods) and possess appliance characteristics (properties). The appliance objects communicate, meaning one object requests the state of another (its properties), commands it to take some action (methods), or requests to be notified when a property of the object changes (an event).

The structure and hierarchy of the AHAM object model is shown below in Figure 2.In Figure 2, an object container is a collection of logically related objects. Also, CHA-1 defines the terms system and subsystem. A system denotes major parts of an appliance. For example, the cook top and the oven are systems of a range. Some parts of a device are considered subsystems. AHAM has developed the object 
hierarchy of Figure 2 for a number of home appliances, including a clothes washer and dryer, refrigerator and freezer, dishwasher, cooking appliances, microwave oven, and room air conditioner.

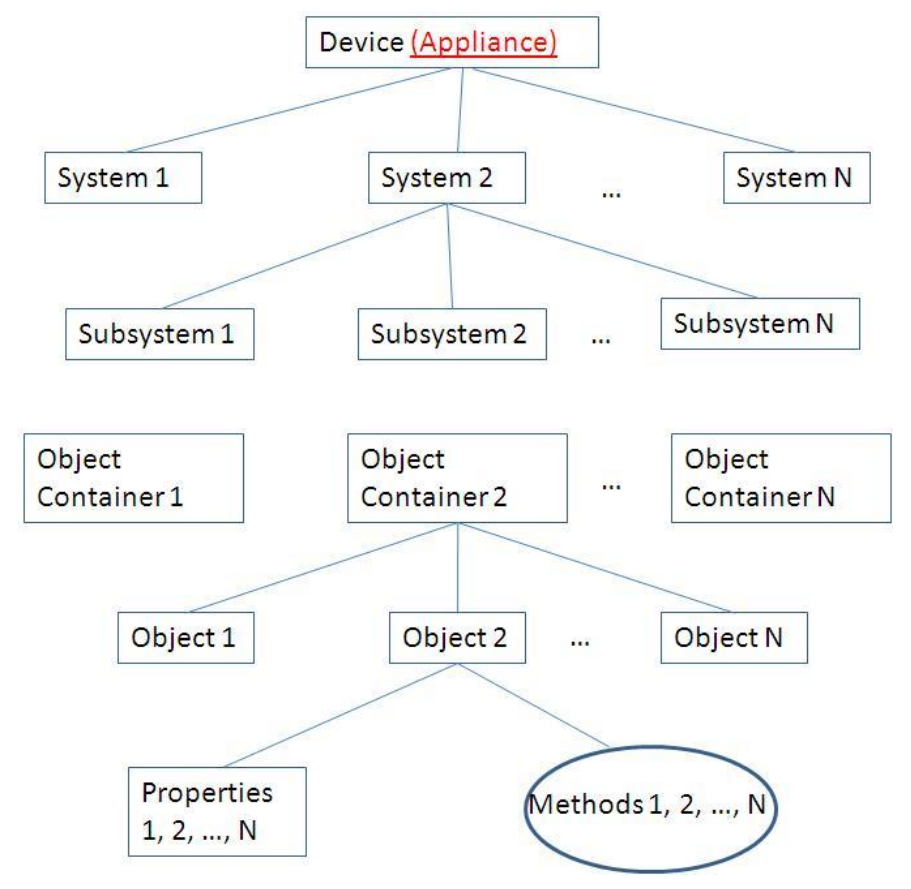

Figure 2. CHA-1 Appliance Model Object Hierarchy (AHAM 2009b)

The standard that AHAM has developed is only an application-layer interoperability standard. No underlying communication protocol, wired or wireless, has been specified. Appliances are accessible and remotely controllable by users, service providers, and other devices, independent of the underlying communication network that is being used. The available functionalities of compliant appliances, as defined by the object model, are transparently accessible through the standard. Each object may be extended with manufacturer-specific features that may or may not be made public.

\subsection{Utility Smart Network Access Port (U-SNAP)}

At present, there exist a number of competing communication protocols and messaging standards (e.g., ZigBee ${ }^{\circledR}$ (ZigBee 2009) and Z-Wave Alliance ${ }^{\circledR}$ (Z-Wave 2009)) that facilitate interaction between smart end-use devices (such as thermostats, in-home displays, pool pumps, water heaters) and a home area network (HAN).

Deployment of millions of smart communicating meters, components of automated metering infrastructure (AMI), continues unabated. Many stakeholders would benefit from a standardized interface between installed AMI components and responsive devices. However, given the number of competing HAN protocols existing in the marketplace today, obtaining consensus agreement to use any one of the protocols is challenging. The mission of the Utility Smart Network Access Port (U-SNAPTM) Alliance is to create a protocol-independent serial-interface standard that accommodates any HAN protocol or messaging standard, present or future (U-SNAP 2009a). The expectation is that such an interface would lead to the unimpeded growth of HAN systems, applications, and services, which, in turn, would enable more rapid growth of demand-response applications. 
In 2007, the California Energy Commission addressed programmable communicating thermostats as part of its Title 24 energy efficiency program. The Commission was considering state-wide demand-response programs as part of planned utility AMI programs. To accomplish this, Radio Data System (RDS) chips (IEC 1999) were installed in every PCT so the thermostats could communicate with AMI smart meters. This posed a challenge in that the PCTs also had to be compatible with the native protocols of AMI meters. It was this challenge that motivated U-SNAP: "Rather than force manufacturers to build and integrate thermostats for each communication protocol deployed, why not offer a simple circuit card that can be "plugged" or "snapped" into a thermostat to connect it to the native AMI network? That way, when one state or utility adopts protocol A and another adopts protocol B, manufacturers can offer the same exact product in multiple markets. The same standard for thermostats can be applied to other HAN devices such as in-home displays, load control modules and even kitchen appliances." (U-SNAP 2009b) The U-SNAP vision is illustrated in Figure 3 below.

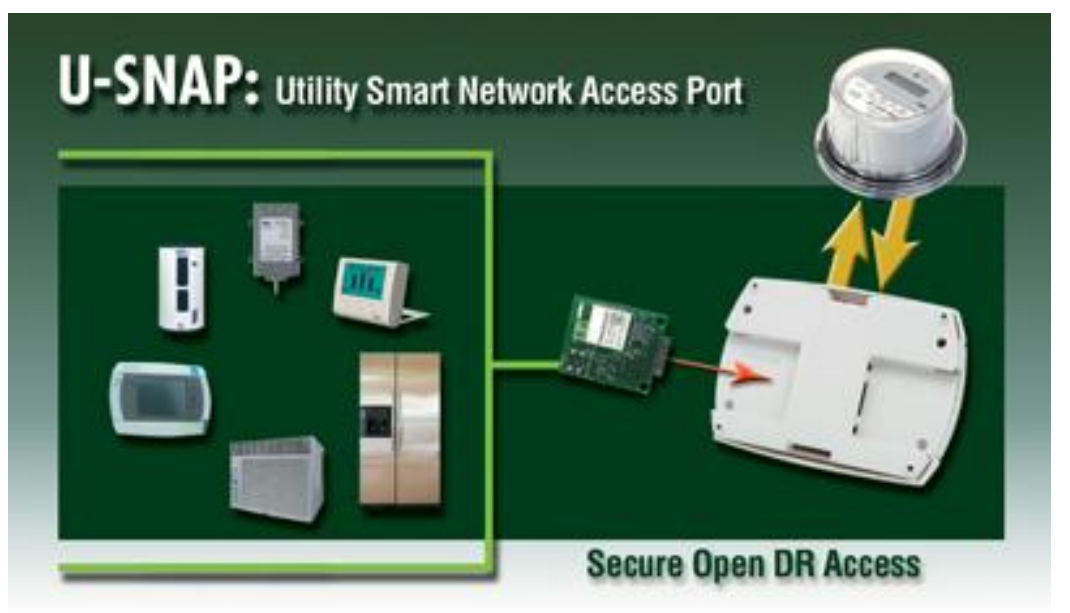

Figure 3. The U-SNAP Vision to Enable Communication between HAN Devices and an AMI Smart Meter is Agnostic of the Underlying Communication or Messaging Protocol (U-SNAP 2009b)

While the U-SNAP vision is an elegant technical solution to facilitate seamless interoperability, what business model would make it attractive for utilities, appliance manufacturers, and consumers to adopt the U-SNAP approach? Typically, adding a communication chip and associated electronics to an appliance would increase product cost on the order of $\$ 10$. Given the low profit margins that may be earned in the manufacture and sale of HAN appliances, this additional expense could prohibit widespread participation of such appliances in demand-response applications. In order to reduce this cost burden for manufacturers, the U-SNAP approach recommends that appliances provide open "U-SNAP" slots. Consumers may then purchase such appliances through traditional retail channels, perhaps incurring only a small incremental cost for the extra U-SNAP connector. Utilities could then ship a pre-configured communication module to their customers. When the customers receive this communication module, they may simply "snap" the module into the open slot of the compatible product. The utility could then connect to, communicate with, and control the device through its U-SNAP interface using an existing AMI network. The U-SNAP alliance estimates that each connector slot and plastic housing could cost less than 10 cents. The U-SNAP card itself is expected to be approximately 1.5 inches square $(3.81 \mathrm{~cm})$, making it small enough to fit into virtually any product (U-SNAP 2009a). The bottom-line goal of USNAP is to provide a very inexpensive interface that enables virtually any consumer product to be connected to a HAN. 
This is the benefit that utilities derive through U-SNAP: if the selection of a communication protocol for responsive devices becomes decoupled in this way from the selection of a communication protocol for the remainder of a HAN system, deployment of HAN products can continue unabated. For manufacturers, the benefit of adopting U-SNAP is that the increment in manufacturing costs is small for appliances that host the inexpensive, generic U-SNAP interface. For consumers, the benefit lies in the realization that no matter which geographical location they move to, and no matter which HAN communication protocols emerge victorious, the responses of their appliances should remain useful and connectable to many HANs.

The U-SNAP business models are not yet complete, according to U-SNAP administrator Barry Hasser (Hasser 2009). It is expected that appliance manufacturers will produce standard products that will operate with or without becoming connected to a U-SNAP communication card. Cards initially are likely to be available from a handful of suppliers, and they could be sourced through utilities, retail suppliers, and other third parties. The small incremental investments necessary to provide the U-SNAP connector and modules are currently being accounted for in one of several ways:

- Some vendors are sharing development costs between a chip supplier and product supplier

- Start-ups are forming to design and produce U-SNAP modules

- Some appliance manufacturers are building their own modules. Currently, Radio Thermostat of America offers U-SNAP cards for a variety of communication protocols, including ZigBee and ZWave (Radio Thermostat 2009). Over time, other appliance makers are likely to offer U-SNAP modules as product accessories.

- Utilities may or may not ultimately bear the incremental expense of providing U-SNAP modules. The outcome may depend on agreements utilities forge with their public-utilities commissions.

The U-SNAP specification (U-SNAP 2009c) defines smart grid devices and U-SNAP communication modules. A smart grid device is a functional element that provides control, monitoring or informational services within a residential or commercial electrical network. A communication module provides communication between an electric utility and its customers and smart grid devices. The root of the specification relies on the serial peripheral interface (SPI) data-transfer-protocol port found on most communication chips, a data-transfer standard that was originally defined by Motorola (U-SNAP 2009c). As emphasized repeatedly, the U-SNAP specification is communication-protocol agnostic. It defines the hardware interface, physical dimensions, data transfer, and message contents. However, various communication modules may be developed to complete the serial communication pathways between U-SNAP-supplied devices and many different serial communication protocols.

There are several U-SNAP cards currently available commercially, and these products support ZigBee $^{\circledR}$, Z-Wave, RDS, WiFi, and Sensus FlexNet ${ }^{\mathrm{TM}}$, and cards for other industry protocols are under development (U-SNAP 2009b). The technical details of the U-SNAP Architecture can be found in (USNAP 2009c). 


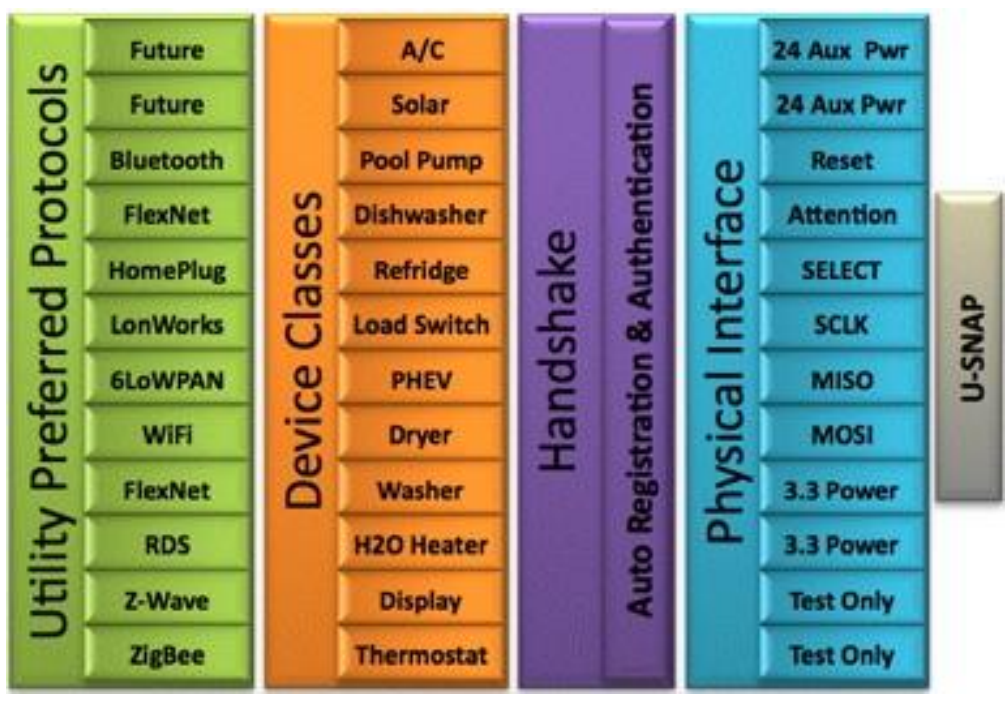

Figure 4. U-SNAP Architecture (U-SNAP 2009b)

The U-SNAP alliance (U-SNAP 2009a) consists of several smart grid stakeholders worldwide, including utilities, AMI suppliers, HAN venders, industry consultants, academics and regulators and has been formed to promote the adoption of the U-SNAP framework. The Electric Power Research Institute recently announced that it will work with the U-SNAP Alliance to develop demand-response-ready appliances (U-SNAP 2009d).

\subsection{Autonomous Control}

Autonomously controlled devices determine and initiate appropriate responses based only upon their independent measurements of available conditions. The observed status of the ac supply voltage, for example, reveals a feeder circuit's voltage and the grid's frequency. These measurements, in turn, allow the inference of useful information about the power grid. Many additional measurements are available for devices to augment or confirm measurements taken from the ac power supply. Autonomously controlled devices are demand-response ready in the sense that they can conduct their tasks and perform their responses without requiring additional communications, connections, or infrastructure.

The Grid Friendly ${ }^{\mathrm{TM}}$ Appliance (GFA) controller is one such autonomous controller. The GFA controller has been designed to fulfill the function of a type of coprocessor chip, able to monitor the power grid and advise appliances of actions that should be taken to help support or stabilize the power grid. The GFA controller was applied to 150 residential clothes dryers and 50 water heaters in the Grid Friendly Appliance Project, during which the GFA controller advised these devices to shed load whenever shallow underfrequency events were encountered on the power grid (Hammerstrom et al. 2007).

In another successful development of autonomously-responsive technology, RLTec of the United Kingdom has initiated placement of frequency-responsive refrigerators in homes across the UK. These refrigerators will provide regulation and spinning-reserve value through the Dynamic Demand program that exists in the UK (Howe 2009). 


\subsection{PinBus}

The PinBus interface approach was based upon the result of collaboration between Whirlpool Corporation, Pacific Northwest National Laboratory (PNNL), and Invensys Controls during the U.S. Department of Energy Grid Friendly ${ }^{\mathrm{TM}}$ Appliance Project (Hammerstrom et al. 2007). In this collaboration, these entities agreed to communicate simple response objectives according to the Boolean voltage states of several pin connectors that were shared among the hardware components belonging to the multiple entities. Recognizing the potential value of this approach, the collaborators jointly wrote and presented the paper (Eustis, Horst and Hammerstrom 2007). This approach was further refined by PNNL and was reported under the name "PinBus" (Hammerstrom 2009).

In this paper, it was argued that many useful responses may be obtained from devices by communicating only high-level grid-management objectives using the voltage states of from one to eight Boolean pins. In fact, PinBus anticipates communication of a broad range of responses beyond the management of system peak load. The interface itself is intended to be identically applied to all devices and device types; however, device manufacturers design their preferred sets of responses and are not obligated to reveal the means by which their devices provide the responses. PinBus communication is bidirectional, allowing acknowledgements and bids to be sent by and received from devices. An interchangeable interface module completes a PinBus system by interpreting between any other communication protocol and the PinBus interface. Ideally, the same interface module may be applied to any device or device type having a PinBus interface Table 1 summarizes the incremental richness of communication features that are supported as the number of device pins grows from one to eight.

Table 1. Capabilities that can be Communicated across the PinBus Interface with Various Numbers of Device Pins (Hammerstrom 2009)

\begin{tabular}{cll}
\hline No. Pins & \multicolumn{1}{c}{ Utility Side } & \multicolumn{1}{c}{ Device Side } \\
\hline 1 & Power curtailment requests & Reveal on/off status \\
2 & Hold power curtailment requests & $\begin{array}{l}\text { Acknowledge power curtailment requests } \\
\text { Reveal override of power curtailment requests }\end{array}$ \\
& & Acknowledge bi-directional real power requests \\
$3-5$ & Bi-directional real power requests & Bid for service using 2-8 discrete levels \\
& Reveal 2-8 price or value levels & Acknowledge bi-directional reactive-power requests \\
6 & Bi-directional reactive-power requests & Alert system / request service \\
\hline
\end{tabular}

Admittedly, the simplicity of PinBus communications means that some functionality cannot be supported. A PinBus interface is not suitable for portals and sensors that necessarily require the communication of rich analog information. A PinBus interface supports the communication of a set of discrete price (or value) levels and bids (e.g., to be interpreted as "price is normal," "price is high," and so on), but a PinBus interface cannot communicate raw price. An optional serial communication pathway might be amended to the PinBus interface for cases where the exchange of rich analog data is necessary.

PinBus development continues at PNNL. A laboratory demonstration is planned for early 2010 to demonstrate a high degree of interoperability at the device, communication-protocol, and use-case levels. 


\subsection{Conclusions}

Slow progress is being made toward the development of devices that are ready to respond to the needs of the electric power grid at the time the devices are purchased. Significant progress has been made toward the development of programmable communicating thermostats that are ready to communicate with smart gateways and meters to control space-conditioning equipment. Major U.S. appliance manufacturers have recently promised to produce and sell lines of responsive, communicating appliances.

Several challenges that delay the production of demand-response-ready devices were listed in this paper. Certainly, significant challenges exist in the areas of standardization, but relatively few purely technical roadblocks have been identified. The business case remains challenging. No competing approach yet presents a complete business case for the production of demand-response-ready devices that would compensate all stakeholders for their investments and risks. The challenge for those who would apply demand-response-ready devices is to define and quantify the benefit that would be achievable from the targeted response and to share that benefit equitably among manufacturers, utility customers, component vendors, device retailers, utilities, and other entities that might benefit from the responses.

Several noteworthy standards and emerging approaches were discussed. Each of these approaches was shown to resolve some of the listed challenges. Several of the strengths and weaknesses of the various approaches are compared below in Table 2.

Table 2. Issues Addressed by the Standards and Approaches Discussed in this Paper

\begin{tabular}{|c|c|c|c|c|c|}
\hline & 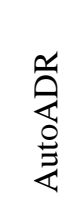 & 志 & 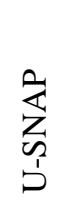 & 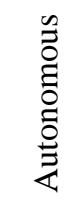 & 表 \\
\hline \multirow{14}{*}{$\begin{array}{l}\text { Addresses both load curtailment and load increases } \\
\text { Addresses fast regulation } \\
\text { Addresses peak reduction } \\
\text { Addresses spinning reserve or balancing services } \\
\text { Applicable to both electrical load and generation } \\
\text { Avoids technology obsolescence } \\
\text { Complete business case that rewards all participants } \\
\text { Facilitates communication protocol interoperability } \\
\text { Facilitates device interoperability } \\
\text { Facilitates use-case interoperability } \\
\text { High level of technology maturity } \\
\text { Inexpensive physical interface } \\
\text { Reduced installation expense } \\
\text { Undifferentiated manufacturing and product distribution }\end{array}$} & 1 & 1 & 1 & 2 & 2 \\
\hline & 0 & 1 & 1 & 2 & 2 \\
\hline & 2 & 2 & 2 & 1 & 2 \\
\hline & 2 & 2 & 2 & 2 & 2 \\
\hline & 1 & 0 & 0 & 2 & 2 \\
\hline & 1 & 2 & 2 & 2 & 2 \\
\hline & 2 & 0 & 1 & 0 & 0 \\
\hline & 2 & 2 & 2 & $\mathrm{n} / \mathrm{a}$ & 2 \\
\hline & $\mathrm{n} / \mathrm{a}$ & 1 & 2 & $\mathrm{n} / \mathrm{a}$ & 2 \\
\hline & 1 & 1 & 1 & $\mathrm{n} / \mathrm{a}$ & 2 \\
\hline & 2 & 1 & 1 & 0 & 0 \\
\hline & 1 & $\mathrm{n} / \mathrm{a}$ & 2 & 2 & 2 \\
\hline & 1 & $\mathrm{n} / \mathrm{a}$ & 2 & 2 & 2 \\
\hline & 0 & 1 & 2 & 1 & 2 \\
\hline
\end{tabular}

0 - Not addressed; 1 - addressed; 2 - strongly addressed; n/a - not applicable 



\subsection{References}

Association of Home Appliance Manufacturers (AHAM). 2009a. "What is AHAM?" Accessed October 12, 2009 online at http://www.aham.org/ht/d/sp/i/329/pid/329.

Association of Home Appliance Manufacturers (AHAM). 2009b. ANSI/AHAM CHA-1-2003.

"Connected Home Appliances-Object Modeling (R2007)." Accessed October 12, 2009 at

http://www.aham.org/ht/d/ProductDetails/sku/4040-140-140/from/714/pid/.

Akuacom 2009. "Automating Demand Response." Akuacom, San Rafael, CA. Accessed October 20, 2009 online at http://www.akuacom.com/index.html.

American National Standards Institute (ANSI). 2009. Accessed October 12, 2009 online at http://www.ansi.org/.

AutomatedBuildings 2009. "Email Interview: Ed Koch \& Ken Sinclair on OpenADR." Automated Buildings. Accessed October 20, 2009 online at http://www.automatedbuildings.com/news/jun09/interviews/090526045707koch.htm.

Eustis C, GR Horst and DJ Hammerstrom. 2007. “Appliance Interface for Grid Responses.” In:

Proceedings of the Grid-Interop Forum, Nov. 7-9, Albuquerque, New Mexico. Accessed December 18, 2009, at http://www.gridwiseac.org/historical/gridinterop2007/default.aspx.

General Electric Company (GE). 2009. "GE Announces Program to Partner with Utilities to Reshape Energy Usage, Help Consumers Save Money.” GE Consumer and Industrial Press Room. General Electric Company, Louisville, Kentucky. Accessed December 12, 2009 online at http://www.geconsumerproducts.com/pressroom/press_releases/company/company/GE_LGE_smartappli ances.htm.

Hammerstrom DJ, J Brous, G Horst, T Oliver, C Eustis, OM Järvegren, RG Pratt, R Kajfasz, P Michie, W Marek, and RL Munson. 2007. Pacific Northwest GridWise ${ }^{\mathrm{TM}}$ Testbed Demonstration Projects; Part II. Grid Friendly Appliance Project. PNNL-17079, Pacific Northwest National Laboratory, Richland, WA. Available at http://gridwise.pnl.gov.

Hammerstrom DJ. 2009. "Pinbus Interface for Interoperable, Grid-Responsive Devices." In Proceedings of the Grid-Interop Forum 2009, November 17-19, 2009, Denver, CO. Soon to be available online at http://www.gridwiseac.org/about/publications.aspx.

Hasser B. 2009. Personal communication with U-SNAP Administrator Barry Hasser on October 15, 2009.

Howe A. 2009. "Dynamic Demand: A Smart Grid Technology." In: Proceedings of the Grid-Interop Forum 2009, November 17-19, 2009, Denver, CO. Accessed December 8, 2009 at http://www.gridinterop.com/2009/\#speaker_1495.

International Electrotechnical Commission (IEC) Subcommittee 100A: Multimedia end-user equipment, of the Technical Committee 100: Audio, video and multimedia systems and equipment. 1999. "Specification of the Radio Data System (RDS) for VHF/FM Sound Broadcasting in the Frequency Range from 87,5 to 108,0 MHZ." RDS Forum. IEC 62106:1999 Standard. RDS Forum Office, Geneva, Switzerland. Available at http://www.rds.org.uk/rds98/pdf/IEC\%2062106-E_no\%20print.pdf. 
Koch E and MA Piette. 2007. "Architecture Concepts and Technical Issues for an Open, Interoperable Automated Demand Response Infrastructure". In Proceedings of the Grid-Interop Forum 2007. GridWise Architecture Council. Accessed October 20, 2009 at http://www.gridwiseac.org/pdfs/forum papers/104 paper final.pdf.

Lawrence Berkeley National Laboratory (LBNL). 2009a. "Automated Demand Response: How the Internet Helps the Electricity Grid in California." LBNL, Berkeley, California. Accessed October 20, 2009 at http://www.lbl.gov/Science-Articles/Archive/sabl/2008/Feb/ADR.html.

Lawrence Berkeley National Laboratory (LBNL). 2009b. "Open Automated Demand Response Communications Specifications Documents." LBNL, Berkeley, California. Accessed October 20, 2009 at http://openadr.lbl.gov/docs.html.

Lawrence Berkeley National Laboratory (LBNL). 2009c. "OpenADR Client Development Program Users Guide". LBNL, Berkeley, CA. Accessed October 20, 2009 at http://openadr.lbl.gov/pdf/openadr-clientdevelop.pdf.

McParland C. 2009. Personal e-mail communication on OpenADR with Charles McParland, Lawrence Berkeley National Laboratory, on October 9, 2009.

Organization for the Advancement of Structured Information Standards (OASIS). 2009. "Organization for the Advancement of Structured Information Standards." OASIS: Advancing Open Standards for the Information Society. Accessed October 20, 2009 at http://www.oasis-open.org/home/.

Radio Thermostat. 2009. "Radio Thermostat Company of America." Radio Thermostat Company of America. Accessed October 20, 2009 at http://www.radiothermostat.com/.

UCA International Users Group (UCAIug). 2009. “UCA International Users Group.” UCAIug. . Accessed October 20, 2009 at http://www.ucaiug.org.

U-SNAP 2009a. “U-SNAP Alliance.” U-SNAP Alliance. Accessed October 9, 2009 at http://www.usnap.org/.

U-SNAP 2009b. "Enabling the Home Area Network Market." U-SNAP Alliance. Accessed October 9, 2009 at http://www.usnap.org/whitePaper.aspx.

U-SNAP 2009c. "U-SNAP Technical Specifications." U-SNAP Alliance. Accessed October 9, 2009 at http://www.usnap.org/technical.aspx.

U-SNAP 2009d. "EPRI and U-SNAP Alliance Establish Liaison Agreement in Effort to Standardize the Interface for Smart Appliances.” U-SNAP Alliance. Press Release November 17, 2009. Accessed December 18, 2009 at http://www.usnap.org/news.aspx.

Whirlpool. 2009. "Whirlpool Corporation to Make All Electronically Controlled Appliances 'Smart Grid' Compatible by 2015." News Release, Whirlpool Corporation, Benton Harbor, Michigan, May 6, 2009. Accessed December 12, 2009 at http://investors.whirlpoolcorp.com/phoenix.zhtml?c=97140\&p=irolnewsArticle \&ID=1285080\&highlight=2015.

ZigBee. 2009. ZigBee Alliance Website. Accessed December 18, 2009 at http://www.Zigbee.org. 
Z-Wave. 2009. "The Z-Wave Alliance Puts Total Control in Your Hands.” Z-Wave Alliance. Accessed October 6, 2009 at http://www.Z-wavealliance.org. 



\section{Distribution}

No. of

Copies

1 Eric Lightner

U.S. Department of Energy

1000 Independence Avenue SW

Washington, DC 20585
No. of

\section{Copies}

\section{Local Distribution}

Pacific Northwest National Laboratory

DJ Hammerstrom

K1-85

CH Imhoff

K1-98

EO Jones

K2-06

RG Pratt

K1-85

CR Sastry

K1-85 


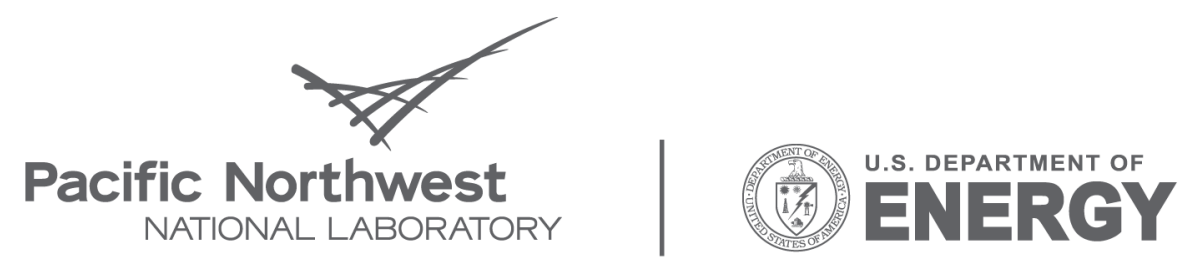

Proudly Operated by Battelle Since 1965

902 Battelle Boulevard

P.O. Box 999

Richland, WA 99352

1-888-375-PNNL (7665)

www.pnl.gov 